Grażyna Horbaczewska, Faculty of Mathematics, Department of Real Functions, Łódź University, Banacha 22, 90-238 Łódź, Poland. e-mail: grhorb@krysia.uni.lodz.pl

\title{
SOME MODIFICATIONS OF THE CORE TOPOLOGY ON THE PLANE
}

\begin{abstract}
In this paper we introduce some modifications of the core topology on the plane. Here the role of the Euclidean topology on the real line on every or almost every direction is played by the density topology or the Hashimoto topology connected with the $\sigma$-ideal of null sets and the $\sigma$-ideal of meager sets. We demonstrate the proper inclusions between these families, we investigate the separation axioms and functions continuous with respect to these topologies.
\end{abstract}

\section{Introduction}

The aim of this paper is to introduce some modifications of the core topology on the plane. The core topology was considered earlier by M. Kuczma and his students $([\mathrm{K}],[\mathrm{KK}])$. The topologies presented here are modifications of this topology, similar to the $d$-crosswise topology (cf. [LMZ], p. 98).

Generally speaking, a point is an interior point of a set $A \subset \mathbb{R}^{2}$ if and only if it is an interior point of the set $A$ in a certain topology on the line in every or $I$-almost every direction, where $I$ is a certain $\sigma$-ideal. Investigations of the topologies of this kind ([WBW], [LS]) can lead one to answers to interesting questions connected with the behavior of real-valued functions on $\mathbb{R}^{2}$ in different directions. The problem of connections between the partial derivatives and directional derivatives was considered by Bruckner and Rosenfeld in [BR].

The comparison of the topologies defined here and already known density topologies seems to be interesting, especially when one considers the open question of the coarsest topology for which strongly approximately continuous

Key Words: Density topologies, core topology, comparison of topologies

Mathematical Reviews subject classification: 54A10 and 28A05

Received by the editors May 9, 1997 
functions are continuous; i.e. of the complete regularity of the topologies and an analogue of the Lusin-Menchoff Theorem ([GNN]).

Recall the notion of the core topology, which is denoted here by $\mathcal{T}^{\prime}$-core. Let $A \subset \mathbb{R}^{2}$. A point $x \in A$ is said to be an algebraically interior point of $A$ if and only if, for each $y \in \mathbb{R}^{2}$, there exists $\varepsilon>0$ such that $x+t y \in A$ for $t \in(-\varepsilon, \varepsilon)$; i.e., $A$ contains an open segment centered at the point $x$ on each line passing through $x$.

The set of all points which are algebraically interior to $A$ is denoted by core $A$. A set $A \subset \mathbb{R}^{2}$ is called algebraically open if $A=$ core $A$. The family

$$
\mathcal{T}^{\prime} \text {-core }=\left\{A \subset \mathbb{R}^{2}: A=\text { core } A\right\}
$$

forms a topology in $\mathbb{R}^{2}$, called the core topology.

There are at least two kinds of natural modifications of this definition; changing the set of directions on which we have a neighborhood in some topology, and changing the topology on the line.

This paper consists of two sections. In the first one we introduce several topologies analogous to the core topology on the plane, using the $\sigma$-ideal of null sets. Here the role of the Euclidean topology on the real line on every or almost every direction is played by the Hashimoto topology or the density topology on the real line. We demonstrate the proper inclusions between these families and compare them with the density topologies and the Hashimoto topology on the plane. We investigate the separation axioms and prove that functions continuous with respect to these topologies are of Baire class 1 .

In the second section we consider analogous problems for Baire category. We obtain a similar diagram for the core topologies connected with the ideal of meager sets. Only the results of the comparison with the ordinary $I$-density topology on the plane and separation axioms are a little bit different. We prove that functions continuous with respect to these topologies are of Baire class at most 2 .

For the terminology and definitions of density point, $I$-density point, density and $I$-density topologies on the line and on the plane $\left(d, d_{I}, d_{2}, d_{I_{2}}, d_{2}^{s}\right.$, $\left.d_{I_{2}}^{s}\right)$, see [CW], [GNN], [PWW], [W], [WB].

Let $m_{k}$ denote Lebesgue measure on $\mathbb{R}^{k}, k=1,2, \mathcal{L}_{k}$ - the family of all measurable sets on $\mathbb{R}^{k}, k=1,2, \mathcal{B}_{k}$ - the family of all sets having the Baire property on $\mathbb{R}^{k}, k=1,2$.

The ball (circle) centered at a point $(x, y)$ with radius $r$ is denoted by $K((x, y), r)(S((x, y), r))$. Let $\sec (\alpha, \beta)$ denote an angular domain determined by the lines forming angles $\alpha$ and $\beta$ with the $x$-axis. Let $p_{\Theta}\left(p_{\Theta}(x)\right), \Theta \in\langle 0, \pi)$, denote the line running through the origin (the point $x$ ) and forming an angle $\Theta$ with the $x$-axis. Let $p_{\Theta}^{+}\left(p_{\Theta}^{+}(x)\right), \Theta \in\langle 0,2 \pi)$, denote the analogous half-line. 
We denote by $\chi_{A}$ the characteristic function of a set $A$ and by $\mathbb{B}_{1}$, Baire class 1 .

\section{Core Topologies Defined with Null Sets}

Definition 2.1. A set $A \subset \mathbb{R}^{2}$ is Hash-core (ap-core) open if and only if, for each point $x \in A$ and for each $\Theta \in\langle 0, \pi)$, the set $p_{\Theta}(x) \cap A$ contains a neighborhood of $x$ in the Hashimoto topology (density topology) on the line $p_{\Theta}(x)$; i.e., a set of the form $G \backslash N$ where $G$ is open in the Euclidean topology, $N$ is a null set and $x \in G \backslash N$. (In other words, $p_{\Theta}(x) \cap A$ contains a linearly measurable set $B$ such that $d_{1}(B, x)=1$ and $x \in B$.)

Let $\mathcal{T}^{\prime}$-Hash-core $\left(\mathcal{T}^{\prime}\right.$-ap-core) denote the family of all Hash-core (ap-core) open sets.

Definition 2.2. A set $A \subset \mathbb{R}^{2}$ is core-a.e. (Hash-core-a.e., ap-core-a.e.) open if and only if, for each point $x \in A$, there exists a linear set $H \subset\langle 0, \pi)$ such that $m_{1}(H)=\pi$ and, for all $\Theta \in H$, the set $p_{\Theta}(x) \cap A$ contains a neighborhood of $x$ in the Euclidean topology (Hashimoto topology, density topology) on the line $p_{\Theta}(x)$.

Let $\mathcal{T}^{\prime}$-core-a.e. $\left(\mathcal{T}^{\prime}\right.$-Hash-core-a.e., $\mathcal{T}^{\prime}$-ap-core-a.e. $)$ denote the family of all core-a.e. (Hash-core-a.e., ap-core-a.e.) open sets.

It is easy to see that the families of sets we have just defined form topologies. They also contain non-measurable sets (see [WBW]).

Theorem 2.3. The following proper inclusions hold.

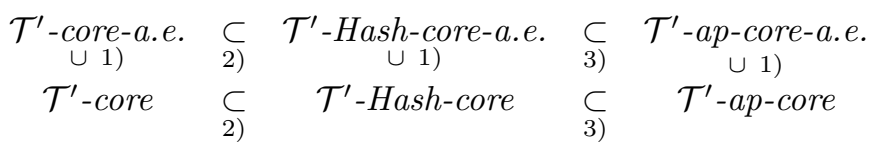

Proof. It suffices to construct three sets. Let

$$
A=\mathbb{R}^{2} \backslash\left\{(x, y) \in \mathbb{R}^{2}: x \neq 0, y=0\right\} .
$$

Then $A \in \mathcal{T}^{\prime}$-core-a.e. $\backslash \mathcal{T}^{\prime}$-ap-core. This shows that the inclusions denoted by 1) are proper. Let $B=\mathbb{R}^{2} \backslash\left(\bigcup_{n \in \mathbb{N}} S\left((0,0), \frac{1}{n}\right)\right)$. Then $B \in \mathcal{T}^{\prime}$-Hash-core $\backslash \mathcal{T}^{\prime}$-core-a.e.

This shows that the inclusions denoted by 2$)$ are proper.

Let $E=\bigcup_{n \in \mathbb{N}}\left(a_{n}, b_{n}\right)$ where $\lim _{n \rightarrow \infty} b_{n}=0,0<a_{n+1}<b_{n+1}<a_{n}$ and 0 is a point of right-hand density of $E$. Let

$$
C=\{(r, \Theta): r \in E, \Theta \in\langle 0,2 \pi)\}
$$


where $(r, \Theta)$ denotes the polar coordinates. Then $C \in \mathcal{T}^{\prime}$-ap-core $\backslash \mathcal{T}^{\prime}$-Hash-core-a.e. This shows that the inclusions denoted by 3) are proper.

To compare the topologies considered with the density topologies on the plane, we assume open sets to be measurable. Thus we define the following families of sets.

$$
\begin{aligned}
& \mathcal{T} \text {-core }=\mathcal{T}^{\prime} \text {-core } \cap \mathcal{L}_{2}, \\
& \mathcal{T} \text {-core-a.e. }=\mathcal{T}^{\prime} \text {-core-a.e. } \cap \mathcal{L}_{2}, \\
& \mathcal{T} \text {-Hash-core }=\mathcal{T}^{\prime} \text {-Hash-core } \cap \mathcal{L}_{2}, \\
& \mathcal{T} \text {-Hash-core-a.e. }=\mathcal{T}^{\prime} \text {-Hash-core-a.e. } \cap \mathcal{L}_{2}, \\
& \mathcal{T} \text {-ap-core }=\mathcal{T}^{\prime} \text {-ap-core } \cap \mathcal{L}_{2}, \\
& \mathcal{T} \text {-ap-core-a.e. }=\mathcal{T}^{\prime} \text {-ap-core-a.e. } \cap \mathcal{L}_{2} .
\end{aligned}
$$

It is easily seen that there are proper inclusions between these families because the sets $A, B, C$ from Theorem 2.3 are measurable.

So we have

$\begin{array}{ccccc}\mathcal{T} \text {-core-a.e. } & \subset & \mathcal{T} \text {-Hash-core-a.e. } & \subset & \mathcal{T} \text {-ap-core-a.e. } \\ \cup & \cup & & \cup \\ \mathcal{T} \text {-core } & \subset & \mathcal{T} \text {-Hash-core } & \subset & \mathcal{T} \text {-ap-core. }\end{array}$

Theorem 2.4. All the families of sets from (1) are contained in the density topology on the plane.

The proof is analogous to that of Theorem 2.4 in [WBW].

Corollary 2.5. Each of the families from (1) forms a topology.

The families from (1) are called core topologies connected with the $\sigma$-ideal of null sets.

Now, we compare them with the Euclidean topology, the Hashimoto topology and the density topologies on the plane.

It is easy to see $([\mathrm{S}])$ that the Euclidean topology is essentially weaker than each of the core topologies connected with the $\sigma$-ideal of null sets.

Theorem 2.6. The strong density topology on the plane is not contained in any of the core topologies connected with the $\sigma$-ideal of null sets. 
Proof. It suffices to prove that $d_{2}^{s} \not \subset \mathcal{T}$-ap-core-a.e. Let $\left\{a_{n}\right\}_{n \in \mathbb{N}}$ be a decreasing sequence of positive numbers tending to 0 such that $\frac{a_{n+1}}{a_{n}} \underset{n \rightarrow \infty}{\longrightarrow} 0$. We construct a set $A$ which is a union of parts of ring-shaped domains with radii $a_{n}$ and $2 a_{n}$. Let $\alpha=\frac{\pi}{4}$. Put

$$
\begin{aligned}
A_{n}^{(k, l)}= & \left\{(r, \Theta): r \in\left\langle a_{\left(2^{n}-n-1\right)+\left(2^{k-1}-1\right)+l} 2 a_{\left(2^{n}-n-1\right)+\left(2^{k-1}-1\right)+l}\right\rangle,\right. \\
\Theta \in & \left\langle\frac{1}{n+2-k} \alpha+\frac{(l-1)\left(\frac{1}{n+1-k}-\frac{1}{n+2-k}\right) \alpha}{2^{k-1}}\right) \alpha \\
& \left.\left.\frac{1}{n+2-k} \alpha+\frac{l\left(\frac{1}{n+1-k}-\frac{1}{n+2-k}\right) \alpha}{2^{k-1}}\right\rangle\right\}
\end{aligned}
$$

for $n \in \mathbb{N}, k \in\{1, \ldots, n\}, l \in\left\{1, \ldots, 2^{k-1}\right\}$ and

$$
A^{0}=\bigcup_{n=1}^{\infty} \bigcup_{k=1}^{n} \bigcup_{l=1}^{2^{k-1}} A_{n}^{(k, l)} \text {. }
$$

We arrange the sets $A_{n}^{(k, l)}$ in a sequence $\left\{C_{m}\right\}_{m \in \mathbb{N}}$ in the following way. $A_{n_{1}}^{\left(k_{1}, l_{1}\right)}$ precedes $A_{n_{2}}^{\left(k_{2}, l_{2}\right)}$ in this sequence if and only if $n_{1}<n_{2}$ or $n_{1}=n_{2}$ and $k_{1}<k_{2}$ or $n_{1}=n_{2}, k_{1}=k_{2}$ and $l_{1}<l_{2}$. So the set $C_{m}$ is a part of the ring with radii $a_{m}$ and $2 a_{m}$. Angles which form the lines marking the set $C_{m}$ with the $x$-axis are denoted by $\alpha_{m}$ and $\beta_{m}, 0<\alpha_{m}<\beta_{m}<\frac{\pi}{4}$. Let $\omega_{m}=\beta_{m}-\alpha_{m}$, $m \in \mathbb{N}$. From the construction it follows that the sequence $\left\{\omega_{m}\right\}_{m \in \mathbb{N}}$ tends to zero.

Put

$$
\begin{aligned}
& A^{1}=\left\{(x, y):(y, x) \in A^{0}\right\} \\
& A=\left\{(x, y):(x, y) \in A^{0} \cup A^{1} \text { or }(-x, y) \in A^{0} \cup A^{1}\right. \\
&\text { or } \left.(x,-y) \in A^{0} \cup A^{1} \text { or }(-x,-y) \in A^{0} \cup A^{1}\right\}
\end{aligned}
$$

and $B=\mathbb{R}^{2} \backslash A$.

Observe that $B$ is not open in the $\mathcal{T}$-ap-core-a.e. topology. Indeed, for each $\Theta \in\langle 0, \pi)$, the set $p_{\Theta} \cap B$ does not contain a neighborhood of the point $(0,0)$ in the density topology on the line because, on the line $p_{\Theta}$, there are intervals of the form $\left(a_{m}, 2 a_{m}\right)$ arbitrarily close to $(0,0)$ and contained in $A$.

We shall show that $B$ is open in the strong density topology on the plane. It suffices to prove that $d_{2}^{s}(B,(0,0))=1$; i.e., for each pair of sequences of positive numbers $\left\{t_{n}\right\}_{n \in \mathbb{N}} \searrow 0$ and $\left\{h_{n}\right\}_{n \in \mathbb{N}} \searrow 0$, we have

$$
d_{n}=\frac{m_{2}\left(B \cap\left(\left\langle-t_{n}, t_{n}\right\rangle \times\left\langle-h_{n}, h_{n}\right\rangle\right)\right)}{4 t_{n} h_{n}} \underset{n \rightarrow \infty}{\longrightarrow} 1 .
$$


We need only show that any subsequence of $\left\{d_{n}\right\}_{n \in \mathbb{N}}$ includes a subsequence tending to 1 . Let us take an arbitrary subsequence $\left\{n_{m}\right\}_{m \in \mathbb{N}}$ of the sequence of positive integers. There are three cases.

$1^{0}$ The sequence $\left\{\frac{t_{n_{m}}}{h_{n_{m}}}\right\}_{m \in \mathbb{N}}$ includes a subsequence $\left\{\frac{t_{n_{m_{p}}}}{h_{n_{m_{p}}}}\right\}_{p \in \mathbb{N}}$ converging to a number $a, 0<a<\infty$. Then there exists a number $\alpha$ such that $0<\alpha<$ $\frac{t_{n_{m_{p}}}}{h_{n_{m_{p}}}}<\frac{1}{\alpha}<\infty$ for sufficiently large $p \in \mathbb{N}$. Hence the sequence of rectangles $E_{n_{m_{p}}}=\left\langle-t_{n_{m_{p}}} t_{n_{m_{p}}}\right\rangle \times\left\langle-h_{n_{m_{p}}} h_{n_{m_{p}}}\right\rangle, p \in \mathbb{N}$, is regular. Consequently, we only need to consider the limit

$$
\lim _{r \rightarrow 0^{+}} \frac{m_{2}(A \cap K((0,0), r))}{\pi r^{2}} .
$$

Fix $r \in\left\langle a_{n}, a_{n-1}\right)$. Then $m_{2}\left(C_{n}\right)=\frac{3}{2} a_{n}^{2} \omega_{n}$ and

$$
\begin{aligned}
\frac{m_{2}(A \cap K((0,0), r))}{\pi r^{2}} & \leq \frac{8 \frac{3}{2} a_{n}^{2} \omega_{n}+\pi\left(2 a_{n+1}\right)^{2}}{\pi r^{2}} \\
& \leq \frac{12}{\pi} \omega_{n}+\frac{4 a_{n+1}^{2}}{a_{n}^{2}}
\end{aligned}
$$

So

$$
\frac{m_{2}(A \cap K((0,0), r))}{\pi r^{2}} \underset{r \rightarrow 0^{+}}{\longrightarrow} 0 .
$$

$2^{0}$ The sequence $\left\{\frac{t_{n_{m}}}{h_{n_{m}}}\right\}_{m \in \mathbb{N}}$ includes a subsequence $\left\{\frac{t_{n_{m_{p}}}}{h_{n_{m_{p}}}}\right\}_{p \in \mathbb{N}}$ tending to $\infty$ and $1^{0}$ does not hold.

Fix $t_{n_{m_{p}}} \in\left\langle a_{k_{p}} a_{k_{p}-1}\right)$. Then, after elementary calculations, we obtain

$$
\begin{aligned}
\left.\frac{m_{2}(A}{A} \cap\left(\left\langle-t_{n_{m_{p}}} t_{n_{m_{p}}}\right\rangle \times\left\langle-h_{n_{m_{p}}} h_{n_{m_{p}}}\right\rangle\right)\right) \\
4 t_{n_{m_{p}}} h_{n_{m_{p}}} \\
\leq \frac{4 \cdot 2 a_{k_{p}+1} h_{n_{m_{p}}}+4 m_{2}\left(C_{k_{p}} \cap\left(\left\langle-t_{n_{m_{p}}} t_{n_{m_{p}}}\right\rangle \times\left\langle-h_{n_{m_{p}}} h_{n_{m_{p}}}\right\rangle\right)\right)}{4 t_{n_{m_{p}}} h_{n_{m_{p}}}} \\
\quad=\frac{2 a_{k_{p}+1}}{t_{n_{m_{p}}}}+\frac{m_{2}\left(C_{k_{p}} \cap\left(\left\langle-t_{n_{m_{p}}} t_{n_{m_{p}}}\right\rangle \times\left\langle-h_{n_{m_{p}}} h_{n_{m_{p}}}\right\rangle\right)\right)}{t_{n_{m_{p}}} h_{n_{m_{p}}}}
\end{aligned}
$$

SO,

$$
\frac{m_{2}\left(A \cap\left(\left\langle-t_{n_{m_{p}}} t_{n_{m_{p}}}\right\rangle \times\left\langle-h_{n_{m_{p}}} h_{n_{m_{p}}}\right\rangle\right)\right)}{4 t_{n_{m_{p}}} h_{n_{m_{p}}}} \underset{p \rightarrow \infty}{\longrightarrow} 0 .
$$

$3^{0}$ The sequence $\left\{\frac{t_{n_{m}}}{h_{n_{m}}}\right\}_{m \in \mathbb{N}}$ includes a subsequence $\left\{\frac{t_{n_{m_{p}}}}{h_{n_{m_{p}}}}\right\}_{p \in \mathbb{N}}$ converging to 0 and neither $1^{0}$ nor $2^{0}$ holds. 
Similar to $2^{0}$, we have

$$
\frac{m_{2}\left(A \cap\left(\left\langle-t_{n_{m_{p}}} t_{n_{m_{p}}}\right\rangle \times\left\langle-h_{n_{m_{p}}} h_{n_{m_{p}}}\right\rangle\right)\right)}{4 t_{n_{m_{p}}} h_{n_{m_{p}}}} \underset{p \rightarrow \infty}{\longrightarrow} 0 .
$$

So, we have proved that $B \in d_{2}^{s} \backslash \mathcal{T}$ ap-core-a.e.

Corollary 2.7. The density topology on the plane is not contained in any of the core topologies connected with the $\sigma$-ideal of null sets.

Theorem 2.8. None of the core topologies connected with the $\sigma$-ideal of null sets is contained in the strong density topology on the plane.

Proof. It is sufficient to show that $\mathcal{T}$-core $\not \subset d_{2}^{s}$. Let

$$
A=\mathbb{R}^{2} \backslash\left\{(x, y) \in \mathbb{R}^{2}: x>0, x^{4} \leq y \leq x^{2}\right\} .
$$

We have

$$
\frac{m_{2}\left(A^{\prime} \cap\left(\left\langle-\frac{1}{n}, \frac{1}{n}\right\rangle \times\left\langle-\frac{1}{n^{4}} \frac{1}{n^{4}}\right\rangle\right)\right)}{m_{2}\left(\left\langle-\frac{1}{n}, \frac{1}{n}\right\rangle \times\left\langle-\frac{1}{n^{4}} \frac{1}{n^{4}}\right\rangle\right)}=\frac{12 n-10}{15 n \cdot 4} .
$$

Consequently, $\overline{d_{2}^{s}}\left(A^{\prime},(0,0)\right)>0$; so $A \notin d_{2}^{s}$. Obviously, $A \in \mathcal{T}$-core. Hence $A \in \mathcal{T}$-core $\backslash d_{2}^{s}$.

Corollary 2.9. None of the core topologies connected with the $\sigma$-ideal of null sets are contained in $d \times d$.

Proof. We have $d \times d \subset d_{2}^{s}$ (see [WB]); so the proof is obvious.

Corollary 2.10. The core topologies connected with the $\sigma$-ideal of null sets and the strong density topology are incomparable.

It is known ([WBW], Th. 2.4) that the topology $d \times d$ is weaker than the $\mathcal{T}$-ap-core topology.

Corollary 2.11. The topology $d \times d$ is weaker than the $\mathcal{T}$-ap-core-a.e. topology.

Theorem 2.12. The topology $d \times d$ is contained in neither the $\mathcal{T}$-Hash-corea.e. topology nor the $\mathcal{T}$-core, $\mathcal{T}$-core-a.e., $\mathcal{T}$-Hash-core.

Proof. It is sufficient to show that $d \times d \not \subset \mathcal{T}$-Hash-core-a.e. Let $E=$ $\bigcup_{n \in \mathbb{N}}\left(a_{n}, b_{n}\right) \cup(-\infty ; 0\rangle$ where $\bigcup_{n \in \mathbb{N}}\left(a_{n}, b_{n}\right)$ is an interval set such that $d(E, 0)=$ 1. Put $A=E \times \mathbb{R}$. Then $A \in d \times d \backslash \mathcal{T}$-Hash-core-a.e.

Theorem 2.13. None of the core topologies connected with the $\sigma$-ideal of null sets is contained in the Hashimoto topology on the plane $\left(\mathcal{O}^{*}\right)$. 
Proof. It follows from Theorem 2.8 because $\mathcal{O}^{*} \subset d_{2}^{s}$.

Theorem 2.14. The Hashimoto topology on the plane is weaker than the $\mathcal{T}$ Hash-core-a.e. and the $\mathcal{T}$-ap-core-a.e. topologies.

Proof. It is sufficient to show that $\mathcal{O}^{*} \subset \mathcal{T}$-Hash-core-a.e. Let $A \in \mathcal{O}^{*}$ and $\left(x_{0}, y_{0}\right) \in A$. We may assume that $x_{0}=y_{0}=0$. We prove that $(0,0)$ is an interior point of $A$ in the $\mathcal{T}$-Hash-core-a.e. topology. Since $A \in \mathcal{O}^{*}$, there exist sets $G, N \subset \mathbb{R}^{2}$ such that $A=G \backslash N, G$ is open in the Euclidean topology on the plane and $m_{2}(N)=0$. By Fubini's theorem for polar coordinates, there exists a set $H \subset\langle 0, \pi)$ such that $m_{1}(H)=0$ and, for $\Theta \in\langle 0, \pi) \backslash H$, the set $N_{\Theta}=N \cap p_{\Theta}$ is a null set on the line $p_{\Theta}$. Consequently, we can choose an open interval $\left(a_{\Theta}, b_{\Theta}\right) \subset G \cap p_{\Theta}$ such that $(0,0) \in\left(a_{\Theta}, b_{\Theta}\right) \backslash N_{\Theta} \subset p_{\Theta} \cap A$ and $m_{1}\left(N_{\Theta}\right)=0$ for almost all $\Theta \in\langle 0, \pi)$. Thus $(0,0)$ belongs to the interior of $A$ in the $\mathcal{T}$-Hash-core-a.e. topology.

Theorem 2.15. The Hashimoto topology on the plane is not contained in any of the topologies: $\mathcal{T}$-core, $\mathcal{T}$-core-a.e., $\mathcal{T}$-Hash-core, $\mathcal{T}$-ap-core.

Proof. It is sufficient to show that $\mathcal{O}^{*} \not \subset \mathcal{T}$-core-a.e. and $\mathcal{O}^{*} \not \subset \mathcal{T}$-ap-core. The set $B$ from Theorem 2.3 is open in $\mathcal{O}^{*}$ and is not open in the $\mathcal{T}$-core-a.e. topology. The set $A$ from the same theorem is open in $\mathcal{O}^{*}$ and is not open in the $\mathcal{T}$-ap-core topology.

The results of Theorems and Corollaries 2.4-2.15 are presented in the following scheme.

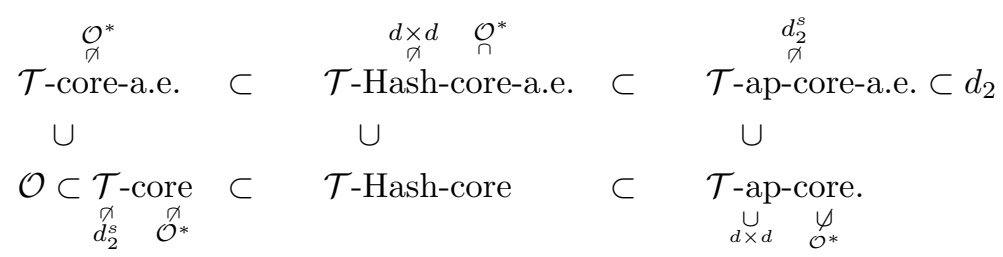

Theorem 2.16. The $\mathcal{T}$-core topology is not regular.

Proof. We show that there exists a set $F$ closed in the $\mathcal{T}$-core topology, which cannot be separated from the point $(0,0)$.

Consider a sequence of rectangles $Q_{k, l}$ where $k \in \mathbb{N} \cup\{0\}, l \in\left\{1, \ldots, 2^{k}\right\}$ and $Q_{k, l}=\left(\frac{l-1}{2^{k}} \frac{l}{2^{k}}\right) \times\left(\frac{1}{2^{k+1}} \frac{1}{2^{k}}\right)$. Form a sequence $\left\{P_{n}\right\}_{n \in \mathbb{N}}$ by putting $P_{n}=Q_{k, l}$ where $n=\sum_{i=0}^{k-1} 2^{i}+l$. Choose points $p_{n} \in P_{n}, n \in \mathbb{N}$, in such a way that any three of them are not colinear. Let $F=\left\{p_{n}: n \in \mathbb{N}\right\}$. Then $F^{\prime}=\mathbb{R}^{2} \backslash F \in \mathcal{T}$-core and $(0,0) \notin F$. 
Let $G, G_{0} \in \mathcal{T}$-core, $(0,0) \in G_{0}$ and $F \subset G$. We shall show that $G \cap G_{0} \neq \emptyset$. The set $G_{0}$ is a neighborhood of $(0,0)$ in the $\mathcal{T}$-core topology; so there exists a number $d \in(0,1)$ such that $\langle 0, d) \times\{0\} \subset G_{0}$. Similarly, $G$ is a neighborhood of the points $p_{n}=\left(x_{n}, y_{n}\right)$ in the $\mathcal{T}$-core topology; so there exist $\delta_{n} \in(0,1)$, $n \in \mathbb{N}$, such that

$$
\bigcup_{n \in \mathbb{N}}\left(x_{n}-\delta_{n}, x_{n}+\delta_{n}\right) \times\left\{y_{n}\right\} \subset G .
$$

Let

$$
E=\limsup _{n}\left(x_{n}-\delta_{n}, x_{n}+\delta_{n}\right)=\bigcap_{n=1}^{\infty} \bigcup_{m=n}^{\infty}\left(x_{m}-\delta_{m}, x_{m}+\delta_{m}\right) .
$$

From the construction of $F$ it follows that the set $\left\{x_{m}, m \geq n\right\}$ is dense on the interval $(0,1)$ for each $n \in \mathbb{N}$. Consequently, $E$ is residual on this interval. Let $x \in E \cap(0, d)$. Since $(x, 0) \in(0, d) \times\{0\} \subset G_{0}$ and $G_{0} \in \mathcal{T}$-core, there exists a number $h>0$ such that $\{x\} \times\langle 0, h) \subset G_{0}$. Then

$$
(\{x\} \times\langle 0, h)) \cap\left(\left(x_{n}-\delta_{n}, x_{n}+\delta_{n}\right) \times\left\{y_{n}\right\}\right) \neq \emptyset
$$

for infinitely many $n \in \mathbb{N}$ (since $x \in\left(x_{n}-\delta_{n}, x_{n}+\delta_{n}\right)$ and $y_{n}<h$ for almost all $n \in \mathbb{N}$ because $\left.y_{n} \underset{n \rightarrow \infty}{\longrightarrow} 0\right)$. Hence $G_{0} \cap G \neq \emptyset$.

Theorem 2.17. The $\mathcal{T}$-Hash-core topology is not regular.

Proof. Let $F=\bigcup_{w \in \mathbb{Q}_{+}} S((0,0), w)$. Obviously, $(0,0) \notin F$ and $F$ is closed in the $\mathcal{T}$-Hash-core topology.

Let $G, G_{0} \in \mathcal{T}$-Hash-core, $(0,0) \in G_{0}$ and $F \subset G$. We shall show that, for each $\Theta \in\langle 0, \pi)$, the intersection $G_{0} \cap G \cap p_{\Theta}$ is non-empty.

Indeed, for each $\Theta \in\langle 0, \pi)$, the set $G \cap p_{\Theta}$ includes, with each point of the set $F \cap p_{\Theta}$, a set of full measure on some interval. Since there are points of the set $F \cap p_{\Theta}$ arbitrarily close to $(0,0)$, the set $G_{0} \cap p_{\Theta}$, as a set containing a neighborhood of $(0,0)$ in the Hashimoto topology on the line $p_{\Theta}$, is not disjoint from $G \cap p_{\Theta}$.

We shall show that the $\mathcal{T}$-Hash-core-a.e. topology is not regular. We shall use the following lemmas.

Lemma 2.18. If $G \in \mathcal{T}$-Hash-core-a.e. and $(0,0) \in G$, then, for each $r>0$,

$$
A_{r}=\left\{\Theta \in\langle 0, \pi): m_{1}\left(G \cap K((0,0), r) \cap p_{\Theta}\right)=2 r\right\}
$$

is measurable. 
The proof follows immediately from the theorem on integration by substitution and Fubini's theorem.

Lemma 2.19. If $G \in \mathcal{T}$-Hash-core-a.e. and $(0,0) \in G$, then there exists a number $n_{0} \in \mathbb{N}$ such that $m_{1}\left(A_{\frac{1}{n_{0}}}\right)>0$, where the sets $A_{r}$ are as above.

Theorem 2.20. The $\mathcal{T}$-Hash-core-a.e. topology is not regular.

Proof. Put $F=\bigcup_{n \in \mathbb{N}} S\left((0,0), \frac{1}{n}\right)$. Obviously, $(0,0) \notin F$ and $F$ is closed in the $\mathcal{T}$-Hash-core-a.e. topology. Let $G, G_{0} \in \mathcal{T}$-Hash-core-a.e., $(0,0) \in G_{0}$ and $F \subset G$. We assume that $G \cap G_{0}=\emptyset$. By Lemma 2.19 , there exists a number $n_{0} \in \mathbb{N}$ such that

$$
A_{\frac{1}{n_{0}}}=\left\{\Theta \in\langle 0, \pi): m_{1}\left(G_{0} \cap K\left((0,0), \frac{1}{n_{0}}\right) \cap p_{\Theta}\right)=\frac{2}{n_{0}}\right\}
$$

has positive linear Lebesgue measure. Let $\Theta_{0}$ be a density point of $A_{\frac{1}{n_{0}}}$. Let us consider $S\left((0,0), \frac{1}{n_{1}}\right)$ where $n_{1}>n_{0}$. Let $\left(x_{0}, y_{0}\right)$ be one of the points of the intersection $S\left((0,0), \frac{1}{n_{1}}\right) \cap p_{\Theta_{0}}$. We can assume that $x_{0}, y_{0}>0$. In the other cases the proof is analogous. Obviously, $\left(x_{0}, y_{0}\right) \in G$. We shall show that $\left(x_{0}, y_{0}\right)$ is not a dispersion point of $G_{0}$, which is a contradiction.

We shall show that there exists $\alpha>0$ such that $\frac{m_{2}\left(K \cap G_{0}\right)}{m_{2}(K)}>\alpha$ for all the balls $K$ with center at the point $\left(x_{0}, y_{0}\right)$ and radius sufficiently small, which means that $\overline{d_{2}}\left(G_{0},\left(x_{0}, y_{0}\right)\right)>0$.

Let $0<a<\frac{1}{n_{0} n_{1}}$. We denote by $\left(x^{\prime \prime}, y^{\prime \prime}\right)$ the point of intersection of the circle $S\left((0,0), \frac{1}{n_{1}}+\frac{a}{2}\right)$ and the half-line $p_{\Theta_{0}}^{+}$, by $\left(x_{2}, y_{2}\right)$, the point of intersection of the circle $S\left(\left(x_{0}, y_{0}\right), a\right)$ and the half-line $p_{\frac{\pi}{2}+\Theta_{0}}^{+}\left(x^{\prime \prime}, y^{\prime \prime}\right)$. Let $\delta$ denote an angle between the half-line $p_{\Theta_{0}}^{+}$and the half-line running from the point $(0,0)$ through the point $\left(x_{2}, y_{2}\right)$. Then

$$
\tan \delta=\frac{\frac{\sqrt{3}}{2} a}{\frac{1}{n_{1}}+\frac{a}{2}} .
$$

Thus $\lim _{a \rightarrow 0} \delta=0$. Put $r_{2}=\sqrt{x_{2}^{2}+y_{2}^{2}}$. Denote by $\left(x^{\prime}, y^{\prime}\right)$ the point of intersection of the circle $S\left((0,0), \frac{1}{n_{1}}-\frac{a}{2}\right)$ and the half-line $p_{\Theta_{0}}^{+}$, by $\left(x_{1}, y_{1}\right)$, the point of intersection of the half-lines $p_{\Theta_{0}+\delta}^{+}$and $p_{\Theta_{0}+\frac{\pi}{2}}^{+}\left(x^{\prime}, y^{\prime}\right)$. Put $r_{1}=\sqrt{x_{1}^{2}+y_{1}^{2}}$.

Then $\cos \delta=\frac{\frac{1}{n_{1}}-\frac{a}{2}}{r_{1}}=\frac{\frac{1}{n_{1}}+\frac{a}{2}}{r_{2}}$. Let

$$
\begin{aligned}
& A=\left(r_{1}, r_{2}\right) \times\left(A_{\frac{1}{n_{0}}} \cap\left(\Theta_{0}-\delta, \Theta_{0}+\delta\right)\right), \\
& U=\left(r_{1}, r_{2}\right) \times\left(\Theta_{0}-\delta, \Theta_{0}+\delta\right) .
\end{aligned}
$$


Obviously, $A \subset U$.

Let $f(r, \Theta)=(r \cos \Theta, r \sin \Theta),(r, \Theta) \in U$. Put $K=K\left(\left(x_{0}, y_{0}\right), a\right)$. Then $f(U) \subset K$ and

$$
\frac{m_{2}(f(U))}{m_{2}(K)}=\frac{\left(r_{2}^{2}-r_{1}^{2}\right) \delta}{\pi a^{2}}=\frac{\frac{2}{n_{1}} \delta}{\pi a \cos ^{2} \delta} .
$$

Since $f(U) \supset f(A)$ and $m_{2}(f(A))=m_{2}\left(f(A) \cap G_{0}\right)+m_{2}\left(f(A) \backslash G_{0}\right)$, it follows that

$$
\frac{m_{2}\left(K \cap G_{0}\right)}{m_{2}(K)} \geq \frac{m_{2}(f(A))-m_{2}\left(f(A) \backslash G_{0}\right)}{m_{2}(K)} .
$$

We have

$$
\begin{aligned}
m_{2}(f(A)) & =\int_{f(U)} \chi_{f(A)}(p) d p=\int_{U} \chi_{f(A)}(f(r, \Theta)) r d r d \Theta \\
& =\int_{\Theta_{0}-\delta}^{\Theta_{0}+\delta} \int_{r_{1}}^{r_{2}} \chi_{f(A)}(f(r, \Theta)) r d r d \Theta \geq \int_{A_{\frac{1}{n_{0}}} \cap\left(\Theta_{0}-\delta, \Theta_{0}+\delta\right)} \frac{r_{2}^{2}-r_{1}^{2}}{2} d \Theta \\
& =\frac{r_{2}^{2}-r_{1}^{2}}{2} m_{1}\left(A_{\frac{1}{n_{0}}} \cap\left(\Theta_{0}-\delta, \Theta_{0}+\delta\right)\right)
\end{aligned}
$$

and

$$
\begin{aligned}
m_{2}\left(f(A) \backslash G_{0}\right) & =\int_{f(U)} \chi_{f(A) \backslash G_{0}}(p) d p=\int_{U} \chi_{f(A) \backslash G_{0}}(f(r, \Theta)) r d r d \Theta \\
= & \int_{\Theta_{0}-\delta}^{\Theta_{0}+\delta} \int_{r_{1}}^{r_{2}} \chi_{f(A) \backslash G_{0}}(f(r, \Theta)) r d r d \Theta \\
= & \int_{\left(\Theta_{0}-\delta, \Theta_{0}+\delta\right) \cap A_{\frac{1}{n_{0}}}} \int_{r_{1}}^{r_{2}} 0 r d r d \Theta \\
& +\int_{\left(\Theta_{0}-\delta, \Theta_{0}+\delta\right) \backslash A_{\frac{1}{n_{0}}}} \int_{r_{1}}^{r_{2}} r d r d \Theta \\
\leq & \frac{r_{2}^{2}-r_{1}^{2}}{2} m_{1}\left(\left(\Theta_{0}-\delta, \Theta_{0}+\delta\right) \backslash A_{\frac{1}{n_{0}}}\right) .
\end{aligned}
$$


Thus

$$
\begin{aligned}
\frac{m_{2}\left(K \cap G_{0}\right)}{m_{2}(K)} & \geq \frac{m_{2}(f(A))-m_{2}\left(f(A) \backslash G_{0}\right)}{m_{2}(f(U))} \frac{m_{2}(f(U)}{m_{2}(K)} \\
& \geq \frac{\left(m_{1}\left(\left(\Theta_{0}-\delta, \Theta_{0}+\delta\right) \cap A_{\frac{1}{n_{0}}}\right)-m_{1}\left(\left(\Theta_{0}-\delta, \Theta_{0}+\delta\right) \backslash A_{\frac{1}{n_{0}}}\right)\right)}{\left(r_{2}^{2}-r_{1}^{2}\right) \delta} \\
& \times \frac{\frac{r_{2}^{2}-r_{1}^{2}}{2} \frac{2}{n_{1}} \delta}{\pi a \cos ^{2} \delta} \\
& =\left[\frac{m_{1}\left(\left(\Theta_{0}-\delta, \Theta_{0}+\delta\right) \cap A_{\frac{1}{n_{0}}}\right)}{2 \delta}-\frac{m_{1}\left(\left(\Theta_{0}-\delta, \Theta_{0}+\delta\right) \backslash A_{\frac{1}{n_{0}}}\right)}{2 \delta}\right] \\
& \times \frac{\frac{2}{n_{1}}}{\pi \cos ^{2} \delta} \frac{\delta}{\operatorname{tg} \delta} \frac{\sqrt{3}}{\frac{2}{n_{1}}+a}
\end{aligned}
$$

by (3).

Since $d\left(A_{\frac{1}{n_{0}}}, \Theta_{0}\right)=1$, it follows that

$$
\begin{aligned}
& \lim _{a \rightarrow 0}[\left.\frac{m_{1}\left(\left(\Theta_{0}-\delta, \Theta_{0}+\delta\right) \cap A_{\frac{1}{n_{0}}}\right)}{2 \delta}-\frac{m_{1}\left(\left(\Theta_{0}-\delta, \Theta_{0}+\delta\right) \backslash A_{\frac{1}{n_{0}}}\right)}{2 \delta}\right] \\
& \quad \times \frac{\frac{2}{n_{1}}}{\pi \cos ^{2} \delta} \frac{\delta}{\operatorname{tg} \delta} \frac{\sqrt{3}}{\frac{2}{n_{1}}+a}=\frac{\sqrt{3}}{\pi}
\end{aligned}
$$

so $\frac{m_{2}\left(G_{0} \cap K\right)}{m_{2}(K)} \geq \frac{\sqrt{3}}{2 \pi}$ for sufficiently small $a$.

Denote by $C_{\mathcal{T}}$ the family of all continuous functions from the plane with a topology $\mathcal{T}$ to the real line with the Euclidean topology. The following inclusions hold.

$$
\begin{aligned}
& C_{\mathcal{T} \text {-core-a.e. }} \quad \subset \quad C_{\mathcal{T} \text {-Hash-core-a.e. }} \quad \subset \quad C_{\mathcal{T} \text {-ap-core-a.e. }} \subset C_{d_{2}} \\
& C_{\mathcal{O}} \subset C_{\mathcal{T} \text {-core }} \quad \subset \quad C_{\mathcal{T} \text {-Hash-core }} \quad \subset \quad C_{\mathcal{T} \text {-ap-core }}
\end{aligned}
$$

Since approximately continuous functions are of Baire class 1, all the functions from the last diagram are also of this class.

Theorem 2.21. The $\mathcal{T}$-core-a.e., $\mathcal{T}$-ap-core and $\mathcal{T}$-ap-core-a.e. topologies are not normal.

Proof. Let $A, B$ be two disjoint countable subsets of $\langle 0,1\rangle \times\{0\}$ which are dense in the Euclidean topology on $\langle 0,1\rangle \times\{0\}$. Then $A$ and $B$ are closed in the $\mathcal{T}$-core-a.e. $(\mathcal{T}$-ap-core, $\mathcal{T}$-ap-core-a.e.) topology. 
We suppose the space to be normal. Then there exists $f \in C_{\mathcal{T} \text {-core-a.e. }}$ $\left(C_{\mathcal{T} \text {-ap-core }}, C_{\mathcal{T} \text {-ap-core-a.e. }}\right)$ such that $f(A)=\{1\}$ and $f(B)=\{0\}$. Consequently, $f$ is discontinuous everywhere on $\langle 0,1\rangle \times\{0\}$. but this is impossible because $f$ is of Baire class 1 .

\section{Core Topologies Defined with Meager Sets}

Analogous to the topologies defined in the previous section we can define the topologies connected with the $\sigma$-ideal of meager sets.

Definition 3.1. A set $A \subset \mathbb{R}^{2}$ is $I$-Hash-core ( $I$-ap-core) open if and only if, for each point $x \in A$ and for each $\Theta \in\langle 0, \pi)$, the set $p_{\Theta}(x) \cap A$ contains a neighborhood of $x$ in the $I$-Hashimoto topology ( $I$-density topology) on the line $p_{\Theta}(x)$; i.e. a set of the form $G \backslash P$ where $G$ is open in the Euclidean topology, $P$ is a linear meager set and $x \in G \backslash P$. (In other words $p_{\Theta}(x) \cap A$ contains a set $B$ having the Baire property, such that $d_{I}(B, x)=1$ and $x \in B$.)

Let $\mathcal{T}^{\prime}-I$-Hash-core $\left(\mathcal{T}^{\prime}-I\right.$-ap-core $)$ denote the family of all $I$-Hash-core $(I-$ ap-core) open sets.

Definition 3.2. A set $A \subset \mathbb{R}^{2}$ is core- $I$-a.e. ( $I$-Hash-core- $I$-a.e., $I$-ap-core- $I$ a.e.) open if and only if, for each $x \in A$, there exists a linear set $H \subset\langle 0, \pi)$ residual on $\langle 0, \pi)$ such that, for all $\Theta \in H$, the set $p_{\Theta}(x) \cap A$ contains a neighborhood of $x$ in the Euclidean topology ( $I$-Hashimoto topology, $I$-density topology) on the line $p_{\Theta}(x)$.

Let $\mathcal{T}^{\prime}$-core- $I$-a.e. $\left(\mathcal{T}^{\prime}\right.$ - $I$-Hash-core- $I$-a.e., $\mathcal{T}^{\prime}$ - $I$-ap-core- $I$-a.e. $)$ denote the family of all core- $I$-a.e. ( $I$-Hash-core- $I$-a.e., $I$-ap-core- $I$-a.e. $)$ open sets.

It is easy to see that the families of sets we have just defined form topologies. They also contain some sets without the Baire property (see [WBW]).

Theorem 3.3. The following proper inclusions hold:

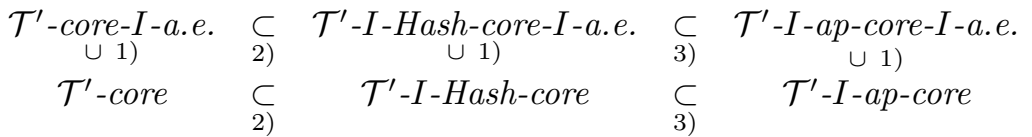

The proof is analogous to that of Theorem 2.3. Only in the last part we take the set $E=\bigcup_{n=\mathbb{N}}^{\infty}\left(a_{n}, b_{n}\right)$ where $0<a_{n+1}<b_{n+1}<a_{n}, \lim _{n \rightarrow \infty} b_{n}=0$ and 0 is a point of right $I$-density of $E$.

To compare the topologies considered here with the $I$-density topologies on the plane, we assume open sets have the Baire property. Thus we define the following families of sets. 


$$
\begin{aligned}
& \mathcal{T}_{I^{-}} \text {-core }=\mathcal{T}^{\prime} \text {-core } \cap \mathcal{B}_{2}, \\
& \mathcal{T} \text {-core- } I \text {-a.e. }=\mathcal{T}^{\prime} \text {-core- } I \text {-a.e. } \cap \mathcal{B}_{2}, \\
& \mathcal{T} \text { - } I \text {-Hash-core }=\mathcal{T}^{\prime} \text { - } I \text {-Hash-core } \cap \mathcal{B}_{2}, \\
& \mathcal{T} \text { - } I \text {-Hash-core- } I \text {-a.e. }=\mathcal{T}^{\prime} \text { - } I \text {-Hash-core- } I \text {-a.e. } \cap \mathcal{B}_{2}, \\
& \mathcal{T} \text { - } I \text {-ap-core }=\mathcal{T}^{\prime} \text { - } I \text {-ap-core } \cap \mathcal{B}_{2}, \\
& \mathcal{T} \text { - } I \text {-ap-core- } I \text {-a.e. }=\mathcal{T}^{\prime} \text { - } I \text {-ap-core- } I \text {-a.e. } \cap \mathcal{B}_{2} .
\end{aligned}
$$

It is easily seen that there are proper inclusions between these families because the sets $A, B, C$ from Theorem 3.3 have the Baire property.

$$
\begin{array}{llclc}
\mathcal{T} \text {-core- } I \text {-a.e. } & \subset & \mathcal{T} \text { - } I \text {-Hash-core- } I \text {-a.e. } & \subset & \mathcal{T} \text { - } I \text {-ap-core- } I \text {-a.e. } \\
\cup & & \cup & & \cup \\
\mathcal{T}_{I} \text {-core } & \subset & \mathcal{T} \text { - } I \text {-Hash-core } & \subset & \mathcal{T} \text { - } I \text {-ap-core. }
\end{array}
$$

Remark 3.4. Each of the families from (4) forms a topology.

The proof is analogous to those of Lemma 2.2 and Theorem 2.3 in [WBW] for the $\mathcal{T}$ - $I$-ap-core family.

The families from (4) will be called core topologies connected with the $\sigma$ ideal of meager sets. Now, we compare them with the Euclidean topology, the $I$-Hashimoto topology and the $I$-density topologies on the plane. It is easy to see that the Euclidean topology is essentially weaker than each of the topologies considered here.

Theorem 3.5. The strong I-density topology is not contained in any of the core topologies connected with the $\sigma$-ideal of meager sets.

Proof. It suffices to prove that $d_{I_{2}}^{s} \not \subset \mathcal{T}$ - $I$-ap-core- $I$-a.e. Consider the set $B$ from Theorem 2.6. It is not open in the $\mathcal{T}$ - $I$-ap-core- $I$-a.e. topology. We shall show that $B$ is open in the strong $I$-density topology on the plane. It suffices to prove that $d_{I_{2}}^{s}\left(A^{0},(0,0)\right)=0$; i.e., for each pair of sequences of positive integers $\left\{n_{m}^{\prime}\right\}_{m \in \mathbb{N}}^{\nearrow},\left\{n_{m}^{\prime \prime}\right\}_{m \in \mathbb{N}}^{\nearrow}$, there exists a subsequence $\left\{m_{p}\right\}_{p \in \mathbb{N}}^{\nearrow}$ such that

$$
\limsup _{p}\left(\left(n_{m_{p}}^{\prime}, n_{m_{p}}^{\prime \prime}\right) A^{0}\right) \cap\langle 0,1\rangle^{2} \in I_{2} .
$$

Let us take any sequence of positive integers $\left\{n_{m}^{\prime}\right\}_{m \in \mathbb{N}}^{\nearrow}$ and $\left\{n_{m}^{\prime \prime}\right\}_{m \in \mathbb{N}}^{\nearrow}$ and fix $m \in \mathbb{N}$. Let $j_{m}$ denote the smallest positive integer for which

$$
C_{j_{m}} \cap\left(\left\langle 0, \frac{1}{n_{m}^{\prime}}\right\rangle \times\left\langle 0, \frac{1}{n_{m}^{\prime \prime}}\right\rangle\right) \neq \emptyset .
$$


Then $\left(\left(n_{m}^{\prime}, n_{m}^{\prime \prime}\right) C_{j_{m}}\right) \cap\langle 0,1\rangle^{2} \neq \emptyset$ and $a_{j_{m}} \leq \frac{\sqrt{2}}{n_{m}^{\prime}}$. Let

$$
\gamma_{m}=\operatorname{arctg}\left(\frac{n_{m}^{\prime \prime}}{n_{m}^{\prime}} \operatorname{tg} \alpha_{j_{m}}\right), \quad \delta_{m}=\operatorname{arctg}\left(\frac{n_{m}^{\prime \prime}}{n_{m}^{\prime}} \operatorname{tg} \beta_{j_{m}}\right) .
$$

Then $\left(n_{m}^{\prime}, n_{m}^{\prime \prime}\right) C_{j_{m}} \subset \sec \left(\gamma_{m}, \delta_{m}\right)$. We shall show that $\delta_{m}-\gamma_{m} \underset{m \rightarrow \infty}{\longrightarrow} 0$.

For an arbitrarily fixed $m \in \mathbb{N}$, let $a$ denote the length of the segment joining the points of intersection of the line $x=n_{m}^{\prime}$ with the $x$-axis and the line $p_{\gamma_{m}}$. Then

$$
a_{m}=n_{m}^{\prime \prime}\left(\operatorname{tg} \beta_{j_{m}}-\operatorname{tg} \alpha_{j_{m}}\right), \quad b_{m}=n_{m}^{\prime \prime} \operatorname{tg} \alpha_{j_{m}} .
$$

By the law of $\operatorname{sines}, \sin \left(\delta_{m}-\gamma_{m}\right)=\frac{a \cos \gamma_{m} \sin \delta_{m}}{a+b}$. Since

$$
\frac{\beta_{m}}{\alpha_{m}} \underset{m \rightarrow \infty}{\longrightarrow} 1, \quad \alpha_{m} \underset{m \rightarrow \infty}{\longrightarrow} 0, \quad \beta_{m} \underset{m \rightarrow \infty}{\longrightarrow} 0
$$

we have $\frac{a_{m}}{b_{m}} \underset{m \rightarrow \infty}{\longrightarrow} 0$; so $\sin \left(\delta_{m}-\gamma_{m}\right) \underset{m \rightarrow \infty}{\longrightarrow} 0$.

Since $0<\delta_{m}<\frac{\pi}{2}$ for each $m \in \mathbb{N}$, we can choose a convergent subsequence $\left\{\delta_{m_{p}}\right\}_{p \in \mathbb{N}}$. Let $\delta_{0}=\lim _{p \rightarrow \infty} \delta_{m_{p}}$. Thus $\delta_{0} \in\left\langle 0, \frac{\pi}{2}\right\rangle$ and

$$
\limsup _{p} \sec \left(\gamma_{m_{p}} \delta_{m_{p}}\right) \subset p_{\delta_{0}} .
$$

Since

$$
\left(n_{m_{p}}^{\prime}, n_{m_{p}}^{\prime \prime}\right) A^{0} \cap\langle 0,1\rangle^{2} \subset\left(\left\langle 0,2 a_{j_{m_{p+1}}} n_{m_{p}}^{\prime}\right\rangle \times\langle 0,1\rangle\right) \cup \sec \left(\gamma_{m_{p}} \delta_{m_{p}}\right),
$$

it follows that

$$
\begin{aligned}
& \limsup _{p}\left(n_{m_{p}}^{\prime}, n_{m_{p}}^{\prime \prime}\right) A^{0} \cap\langle 0,1\rangle^{2} \subset \limsup _{p}\left(\left\langle 0,2 a_{j_{m_{p+1}}} n_{m_{p}}^{\prime}\right\rangle \times\langle 0,1\rangle\right) \\
& \cup \limsup _{p} \sec \left(\gamma_{m_{p}} \delta_{m_{p}}\right) \text {. }
\end{aligned}
$$

From $a_{j_{m_{p}}} n_{m_{p}}^{\prime} \leq \sqrt{2}$ and $\frac{a_{j_{m_{p+1}}}}{a_{j_{m_{p}}}} \underset{p \rightarrow \infty}{\longrightarrow} 0$ we have $2 a_{j_{m_{p+1}}} n_{m_{p}}^{\prime} \underset{p \rightarrow \infty}{\longrightarrow} 0$; so the set $\lim \sup _{p}\left(\left\langle 0,2 a_{j_{m_{p+1}}} n_{m_{p}}^{\prime}\right\rangle \times\langle 0,1\rangle\right)$ is included in the segment $\{0\} \times\langle 0,1\rangle$. Consequently, by (5), $\limsup _{p}\left(\left(n_{m_{p}}^{\prime}, n_{m_{p}}^{\prime \prime}\right) A^{0}\right) \cap\langle 0,1\rangle^{2} \in I_{2}$.

Corollary 3.6. The I-density topology on the plane is not contained in any of the core topologies connected with the $\sigma$-ideal of meager sets. 
Theorem 3.7. None of the core topologies connected with the $\sigma$-ideal of meager sets is contained in the strong I-density topology on the plane.

Proof. It is sufficient to show that $\mathcal{T}_{I^{-}}$core $\not \subset d_{I_{2}}^{s}$. The set $A$ from Theorem 2.8 is not open in the strong $I$-density topology, more precisely, $(0,0)$ is not a strong $I$-density point of $A$. For the sequences $\{n\}_{n \in \mathbb{N}}$ and $\left\{n^{3}\right\}_{n \in \mathbb{N}}$ and any subsequences $\left\{n_{k}\right\}_{k \in \mathbb{N}}$ and $\left\{n_{k}^{3}\right\}_{k \in \mathbb{N}}$, we have $\bigcap_{k \in \mathbb{N}} \bigcup_{l>k}\left[\left(n_{l}, n_{l}^{3}\right) A^{\prime}\right] \cap$ $\langle-1,1\rangle^{2} \supset(0,1\rangle^{2}$. This means that $\limsup _{k}\left[\left(n_{k} n_{k}^{3}\right) A^{\prime}\right] \cap\langle-1,1\rangle^{2} \notin I_{2}$. Hence

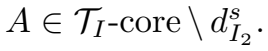

Corollary 3.8. None of the core topologies connected with the $\sigma$-ideal of meager sets is contained in $d_{I} \times d_{I}$.

Proof. We have $d_{I} \times d_{I} \subset d_{I_{2}}^{s}$ (see [WB]); so the proof is obvious.

Corollary 3.9. The core topologies connected with the $\sigma$-ideal of meager sets and the strong I-density topology are incomparable.

It is known (see [WBW], Th. 2.3) that the topology $d_{I} \times d_{I}$ is weaker than the $\mathcal{T}$ - $I$-ap-core topology. Consequently the topology $d_{I} \times d_{I}$ is weaker than the $\mathcal{T}$ - $I$-ap-core- $I$-a.e. topology.

Theorem 3.10. The topology $d_{I} \times d_{I}$ is contained in neither the $\mathcal{T}$-I-Hashcore-I-a.e. nor the $\mathcal{T}_{I}$-core, $\mathcal{T}$-core-I-a.e., $\mathcal{T}$-I-Hash-core.

Proof. It is sufficient to show that $d_{I} \times d_{I} \not \subset \mathcal{T}$-I-Hash-core-I-a.e. Change the set from Theorem 2.13. Let $E=\bigcup_{n \in \mathbb{N}}\left(a_{n}, b_{n}\right) \cup(-\infty, 0\rangle$ where $\bigcup_{n \in \mathbb{N}}\left(a_{n}, b_{n}\right)$ is an interval set such that $d_{I}(E, 0)=1$. Put $A=E \times \mathbb{R}$. Then

$$
A \in d_{I} \times d_{I} \backslash \mathcal{T} \text { - } I \text {-Hash-core- } I \text {-a.e. }
$$

It is also known (see [WBW], Cor. 2.5) that the $\mathcal{T}$ - $I$-ap-core topology is not contained in the $I$-density topology on the plane. Consequently, the $\mathcal{T}-I$-ap-core- $I$-a.e. is not contained in the $I$-density topology on the plane.

Theorem 3.11. The I-density topology on the plane is stronger than the $\mathcal{T}$ $I$-Hash-core-I-a.e., $\mathcal{T}$-core-I-a.e. and $\mathcal{T}_{I}$-core topologies.

Proof. It is sufficient to show that $\mathcal{T}$ - $I$-Hash-core- $I$-a.e. $\subset d_{I_{2}}$. Assume there exists a set $A$ such that $A \in \mathcal{T}$-I-Hash-core- $I$-a.e. and there exists a point $\left(x_{0}, y_{0}\right) \in A$ such that $d_{I_{2}}\left(A,\left(x_{0}, y_{0}\right)\right) \neq 1$. We can assume that $x_{0}=y_{0}=$ 0 . Then there exists a sequence $\left\{n_{k}\right\}_{k \in \mathbb{N}}^{\nearrow}$ of positive integers such that, for any of its subsequences $\left\{n_{k_{m}}\right\}_{m \in \mathbb{N}}^{\nearrow}$, we have $B=\bigcap_{l=1}^{\infty} \bigcup_{m=l}^{\infty}\left(n_{k_{m}} n_{k_{m}}\right) A^{\prime} \cap$ 
$\langle-1,1\rangle^{2} \notin I_{2}$. Obviously, $B$ has the Baire property; so it is residual on some ball $K((x, y), \delta)$. We can assume that $(0,0) \notin K((x, y), \delta)$ and $y>0$.

Let $p_{\Theta_{1}}, p_{\Theta_{2}}$, where $\Theta_{1}<\Theta_{2}$, are lines tangent to the ball $K((x, y), \delta)$ passing through the point $(0,0)$. By the Kuratowski-Ulam theorem ([O], p.56), there exists a set $H_{1} \subset\left(\Theta_{1}, \Theta_{2}\right)$ residual on this interval and such that, for $\Theta \in$ $H_{1}$, the set $B_{\Theta}=K((x, y), \delta) \cap B \cap p_{\Theta}$ is residual on the interval $K((x, y), \delta) \cap$ $p_{\Theta}$. We shall denote this interval by $\left(a_{\Theta}, b_{\Theta}\right)$. Since $A \in \mathcal{T}$ - $I$-Hash-core- $I$-a.e. and $(0,0) \in A$, there exists a set $H_{2} \in\langle 0, \pi)$ residual on this interval and such that, for $\Theta \in H_{2}$, there exist $\varepsilon_{\Theta}>0$ and a set $K_{\Theta} \subset p_{\Theta}$ which is meager on this line and such that $\left(-\varepsilon_{\Theta}, \varepsilon_{\Theta}\right) \backslash K_{\Theta} \subset A \cap p_{\Theta}$.

Let now $\Theta \in H_{1} \cap H_{2}$. We notice that there exists $n_{0} \in \mathbb{N}$ such that $n \varepsilon_{\Theta}>$ $b_{\Theta}$ for any $n>n_{0}$. Since $\left(-\varepsilon_{\Theta}, \varepsilon_{\Theta}\right) \backslash K_{\Theta} \subset A$, we have $\left(a_{\Theta}, b_{\Theta}\right) \backslash n K_{\Theta} \subset n A$ for $n>n_{0}$. Let $p \in\left(a_{\Theta}, b_{\Theta}\right) \cap B \backslash n K_{\Theta}$. Then $p \in\left(a_{\Theta}, b_{\Theta}\right) \cap B=B_{\Theta}$; so $p \in$ $\left(n_{k_{m}}, n_{k_{m}}\right) A^{\prime}$ for infinitely many $m \in \mathbb{N}$, which means that $\left(\frac{1}{n_{k_{m}}}, \frac{1}{n_{k_{m}}}\right) p \notin A$ for infinitely many $m \in \mathbb{N}$.

On the other hand, $p \in\left(a_{\Theta}, b_{\Theta}\right) \backslash n K_{\Theta} \subset n A$ for $n>n_{0}$. Thus $\left(\frac{1}{n}, \frac{1}{n}\right) p \in A$ for each $n>n_{0}$. This contradiction ends the proof.

Theorem 3.12. None of the core topologies connected with the $\sigma$-ideal of meager sets is contained in the I-Hashimoto topology on the plane $\left(\mathcal{O}_{I}^{*}\right)$.

The proof follows from Theorem 3.7 because $\mathcal{O}_{I}^{*} \subset d_{I_{2}}^{s}$.

Theorem 3.13. The I-Hashimoto topology on the plane is weaker than the $\mathcal{T}$-I-Hash-core-I-a.e. and the $\mathcal{T}$-I-ap-core-I-a.e. topologies.

Proof. As in the proof of Theorem 2.15, $\mathcal{O}_{I}^{*} \subset \mathcal{T}$-I-Hash-core- $I$-a.e. by using the Kuratowski-Ulam theorem for the polar coordinates.

Theorem 3.14. The I-Hashimoto topology on the plane is not contained in any of the topologies: $\mathcal{T}_{I}$-core, $\mathcal{T}$-core-I-a.e., $\mathcal{T}$-I-Hash-core, $\mathcal{T}$-I-ap-core.

Proof. It is enough to show that $\mathcal{O}_{I}^{*} \not \subset \mathcal{T}$-core- $I$-a.e. and $\mathcal{O}_{I}^{*} \not \subset \mathcal{T}$-I-ap-core. The set $B$ from Theorem 2.3 is open in $\mathcal{O}_{I}^{*}$ and is not open in the $\mathcal{T}$-core- $I$ a.e. The set $A$ from the same theorem is open in $\mathcal{O}_{I}^{*}$ and is not open in the $\mathcal{T}$ - $I$-ap-core topology.

The results of Theorems and Corollaries 3.5-3.14 are presented in the fol- 
lowing scheme.

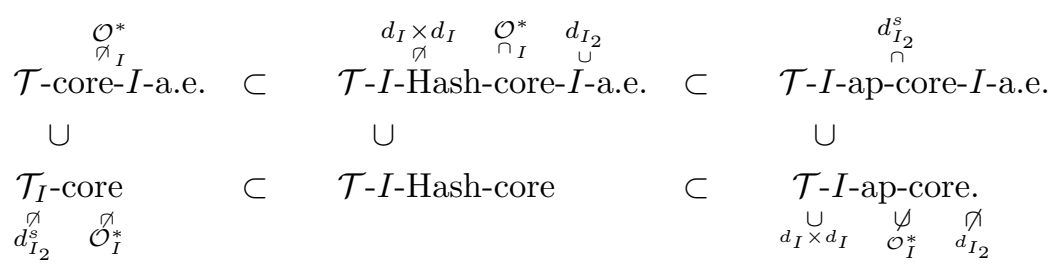

We can see that only the results of the comparison of the $I$-density topologies with the core topologies connected with the $\sigma$-ideal of meager sets differ from the analogous ones obtained in the previous section.

Now, we present theorems on the regularity of the core topologies connected with the $\sigma$-ideal of meager sets.

Theorem 3.15. The $\mathcal{T}_{I}$-core topology is not regular.

The proof is analogous to that of Theorem 2.17.

Theorem 3.16. The $\mathcal{T}$-core-I-a.e. topology is not regular.

Proof. Consider a sequence of sets $Q_{k, l}$ where $k \in \mathbb{N} \cup\{0\}, l \in\left\{1, \ldots, 2^{k}\right\}$ and $Q_{k, l}=\left\{(r, \Theta): r \in\left(\frac{1}{2^{k+1}} \frac{1}{2^{k}}\right), \Theta \in\left(\frac{l-1}{2^{k}} \frac{\pi}{2} \frac{l}{2^{k}} \frac{\pi}{2}\right)\right\}$. Let us form a sequence $\left\{K_{n}\right\}_{n \in \mathbb{N}}$ by putting $K_{n}=Q_{k, l}$ where $n=\sum_{i=0}^{k-1} 2^{i}+l$. Choose points $p_{n} \in K_{n}$, $n \in \mathbb{N}$, in such a way that any three of them are not colinear. Let $F=\left\{p_{n}\right.$ : $n \in \mathbb{N}\}$. Then $F^{\prime}=\mathbb{R}^{2} \backslash F \in \mathcal{T}$-core- $I$-a.e. and $(0,0) \notin F$.

Let $G, G_{0} \in \mathcal{T}$-core- $I$-a.e., $(0,0) \in G_{0}$ and $F \subset G$. We shall show that $G_{0} \cap G \neq \emptyset$. Let $r_{p}, \Theta_{p}$ denote the polar coordinates of $p$. Put $\widehat{p}_{n}=\left(1, \Theta_{p_{n}}\right)$ for $n \in \mathbb{N}$. From the construction of $F$ it follows that the set $\left\{\widehat{p}_{k}: k \geq n\right\}$ is dense on the $\operatorname{arc} L=\left\{(r, \Theta): r=1, \Theta \in\left(0, \frac{\pi}{2}\right)\right\}$. Hence, for each $n \in \mathbb{N}$, the set $\left\{\Theta_{p_{k}}: k \geq n\right\}$ is dense on $\left(0, \frac{\pi}{2}\right)$.

Let $\widehat{G}=\left\{\left(1, \Theta_{p}\right): p \in G\right\}$. Since $G \in \mathcal{T}$-core- $I$-a.e., it contains, with every point $p_{n}$, a neighborhood $A_{n}$ of $p_{n}$ in the $\mathcal{T}$-core- $I$-a.e. topology (we may assume that $A_{n} \subset K_{n}$ ). Then $\widehat{G}$ contains, with every point $\widehat{p}_{n}$, an open arc containing $\widehat{p}_{n}$. We denote that arc by $\widehat{A}_{n}$. Then $\bigcup_{k \geq n} \widehat{A}_{k}$ is residual on $L$ for every $n \in \mathbb{N}$ and so is $\widehat{A}=\lim \sup _{n} \widehat{A}_{n}$. Thus the set $U=\{\Theta:(1, \Theta) \in \widehat{A}\}$ is residual on $\left\langle 0, \frac{\pi}{2}\right)$.

Since $G_{0}$ is a neighborhood of $(0,0)$ in the $\mathcal{T}$-core- $I$-a.e. topology, $G_{0}$ contains neighborhoods of $(0,0)$ in the Euclidean topology on each direction from $\left(0, \frac{\pi}{2}\right) \backslash P$ where $P$ is some set of the first category. 
Let $\Theta \in U \backslash P$. The set $G_{0}$ contains a segment $I_{\Theta}$ with center $(0,0)$, $I_{\Theta} \subset p_{\Theta}$. Since $\Theta \in U$, the line $p_{\Theta}$ intersects infinitely many sets $A_{n}$. Simultaneously, the segment $I_{\Theta}$ is disjoint from a finite quantity of the rings $\left\{(r, \Theta): r \in\left(\frac{1}{2^{k+1}} \frac{1}{2^{k}}\right), \Theta \in\langle 0,2 \pi)\right\}$; so $I_{\Theta}$ intersects infinitely many sets $A_{n}$. Hence $G_{0} \cap G \neq \emptyset$ because $I_{\Theta} \subset G_{0}$ and $A_{n} \subset G$ for each $n \in \mathbb{N}$.

Theorem 3.17. The $\mathcal{T}$-I-Hash-core, $\mathcal{T}$-I-Hash-core-I-a.e., $\mathcal{T}$-I-ap-core and $\mathcal{T}$-I-ap-core-I-a.e. topologies are not regular.

Proof. First consider the $\mathcal{T}$ - $I$-Hash-core topology. Let $F=\bigcup_{w \in \mathbb{Q}_{+}} S((0,0), w)$. Then $F$ is closed in the $\mathcal{T}$ - $I$-Hash-core topology, and $(0,0) \notin F$.

Let $G_{0}, G \in \mathcal{T}$-I-Hash-core. Then they have the Baire property and they are of the second category on the plane. We assume that $(0,0) \in G_{0}$ and $F \subset G$. We shall show that $G \cap G_{0} \neq \emptyset$. The set $G_{0}$ is residual on a certain ball $K$. This ball has common points with $F$ and contains their neighborhoods in the $\mathcal{T}$ - $I$-Hash-core topology, included in $G$ and of the second category on the plane. Thus $G \cap G_{0} \neq \emptyset$.

This proof also shows that the $\mathcal{T}$ - $I$-Hash-core- $I$-a.e., $\mathcal{T}$ - $I$-ap-core and $\mathcal{T}$ - $I$ ap-core- $I$-a.e. topologies are not regular. ( $F$ is closed in these topologies and non-empty open sets have the Baire property and are of the second category on the plane.)

We can consider the families of continuous functions with respect to the core topologies connected with the $\sigma$-ideal of meager sets.

The following inclusions hold.

$$
\begin{array}{ccccc} 
& C_{d_{I_{2}}} & & \\
C_{\mathcal{T} \text {-core- } I \text {-a.e. }} & \subset & C_{\mathcal{T} \text { - } I \text {-Hash-core- } I \text {-a.e. }} & \subset & C_{\mathcal{T} \text { - } \text {-ap-core- } I \text {-a.e. }}^{\cup} \\
C_{\mathcal{T}_{\text {I }} \text {-core }} & \subset & C_{\mathcal{T} \text { - } I \text {-Hash-core }} & \subset & C_{\mathcal{T} \text {-I-ap-core }}
\end{array}
$$

Since $C_{d_{I_{2}}} \subset \mathbb{B}_{1}$ (see [PWW]), the functions belonging to $C_{\mathcal{T}_{I} \text {-core }}, C_{\mathcal{T} \text {-core- } I \text {-a.e. }}$, $C_{\mathcal{T} \text {-I-Hash-core }}, C_{\mathcal{T} \text {-I-Hash-core-I-a.e. }}$ are of Baire class 1 . The functions belonging to $C_{\mathcal{T} \text { - } I \text {-ap-core }}$ are separate $I$-approximately continuous. By Theorem 3.1 [BLW], they are at most of Baire class 2.

\section{References}

[BLW] M. Balcerzak, E. Lazarow, W. Wilczyński, On one- and twodimensional I-densities and related kinds of continuity, Real Analysis Exch., 13 No. 1, (1987-1988), 80-92, 130-150, 120-121. 
[BR] A. Bruckner, M. Rosenfeld, A theorem on approximate directional derivatives, Ann. Seuola Norm. Pisa , 22, (1968), 343-350.

[CW] R. Carrese, W. Wilczyński, I-density points of plane sets, Ricerche di Matematica , 34 No. 1, (1985), 147-157.

[GNN] C. Goffman, C. Neugebauer, T. Nishiura, Density topology and approximate continuity, Duke Math. J., 28, (1961), 497-505.

[H] H. Hashimoto, On the * topology and its applications, Fund. Math., 91, (1976), 5-10.

[K] M. Kuczma, A note on the core topology, Annales Math. Silesianae, $\mathbf{5}$ No. 1236 (1991), 28-36.

[KK] , Z. Kominek, M. Kuczma, Theorems of Bernstein-Doetsch, Piccard and Mehdi, and semilinear topology, Arch. Math. (Basel), 52 , (1989), 595-602.

[LMZ] J. Lukeš, J. Malý, L. Zajiček, Fine topology methods in real analysis and potential theory, LN in Math. 1189, Springer Verlag, New York, 1986.

[LS] I. Libicka, B. Szkopińska, On directional $I_{1}$ - density points, Demonstratio Mathematica, XXX No. 3, (1997), 479-484.

[O] J.C. Oxtoby, Measure and category, Springer Verlag, New York, 1980.

[PWW] W. Poreda, E. Wagner-Bojakowska, W. Wilczyński, A category analogue of the density topology, Fund. Math. , 125, (1985), 167-173.

[S] T. Świạtkowski, Some properties of cross- and core-topologies on $\mathbb{R}^{2}$, Scientific Bulletin of Łódź Technical University, Nr 687, Matematyka, z. $25,(1993), 77-81$.

[W] W. Wilczyński, A category analogue of the density topology, approximate continuity and the approximate derivative, Real Analysis Exch., 10 No. 2 , (1984-85), 241-265.

[WB] E. Wagner-Bojakowska, Rotation invariant density topologies on the plane, Atti Sem. Mat. Fis. Univ. Modena , 42, (1994), 227-254.

[WBW] E. Wagner-Bojakowska, W. Wilczyński, Approximate core topologies, Real Analysis Exch. , 20 No. 1, (1994-95), 192-203 